\title{
Una plataforma de conocimiento intergeneracional e interdisciplinar en la comarca de La Serranía (Valencia)
}

\author{
Francisca Roger Espinosa
}

Univeridad Politècnica de València, España

\begin{abstract}
Resumen
Entre la educación y la investigación, la realidad social y la política representan la experiencia práctica y real de nuestros valores cívicos y ciudadanos. Por tanto, conectar la educación con la investigación, con la sociedad y con la política es un reto dentro de nuestro sistema educativo, sometido a sucesivos cambios que afectan tanto a su modelo estructural como a su modelo envolvente y a su resultante final.

Los trabajos de investigación desarrollados como personal externo al ámbito académico en esa comarca, pero realizados para su aprobación en este entorno, nos han llevado a valorar la necesidad de ensamblar ambos espacios en todas sus direcciones: sociedad, cultura, salud, empleo y economía, entre otros. En nuestro caso, nos centramos en la dirección cultural como eje generador de identidades sociales y territoriales que enlazan los conocimientos del pasado con las estrategias del futuro. En concreto, nos detenemos en el campo del patrimonio cultural $y$, especialmente, dentro de la disciplina arquitectónica como testimonio del arte plástico de valor inmueble presente en nuestro territorio, y como prueba histórica construida por las diferentes civilizaciones que lo han ocupado y lo han transformado en su hábitat.
\end{abstract}

La propuesta del estudio busca poner en relación el proceso educativo con su contexto social y político a través de la participación -cruzada, correctiva, informativa y difusora- dentro del mundo de la educación artística proyectada a lo largo de la vida de las personas: durante todo su proceso educativo, formativo y universitario; durante su etapa laboral, productiva y profesional; durante su veteranía experimentada, contrastada y probada. Se planean prácticas autónomas y grupales intergeneracionales, se 
Una plataforma de conocimiento intergeneracional e interdisciplinar en la comarca de La Serranía (Valencia)

proyectan actividades dentro de la educación formal e informal con patrocinio empresarial y se diseña una plataforma de conocimiento compartido y colaborativo como nexo de unión entre todos los agentes intervinientes.

Palabras clave: Educación, investigación, sociedad, interdisciplinariedad.

\section{Introducción}

En la comarca de La Serranía, una zona de interior, situada al noroeste de la provincia de Valencia, hemos centrado nuestra labor investigadora a lo largo de los últimos años con el propósito de fortalecer el aprecio hacia el patrimonio cultural reconocido al amparo de la legislación vigente -estatal y autonómica- y, a su vez, poner en valor el patrimonio cultural no reconocido, que subyace en el sentir social de su ciudadanía. En este sentido, el proceso de participación ciudadana se ha iniciado desde el ámbito de la educación secundaria y, ahora, se ha planificado su continuidad hasta dar voz a todos los segmentos de población que actualmente viven y participan activamente en el desarrollo cultural de la comarca. Por ello, hablamos de conocimiento intergeneracional -entre niños, adolescentes, jóvenes, adultos y mayores-, así como de conocimiento interdisciplinar -entre oficios, empresas, administraciones y entidades- que envuelven la estructura humana de esta comunidad comarcal.

\section{Objetivo}

El objetivo de este trabajo es diseñar, crear y alimentar una plataforma virtual informativa en la que se muestren los diferentes caminos de búsqueda que nos devuelven la construcción del conocimiento cultural de la comarca y de sus pueblos, más allá de sus límites y desde el propio territorio. Es decir, con el aprovechamiento de las nuevas tecnologías y de los nuevos recursos empleados en el tratamiento de la información documental, escrita, gráfica, fotográfica y sonora, se pretende mantener la transversalidad intergeneracional e interdisciplinar, desde dentro y desde fuera del lugar $\mathrm{y}$, tejiendo una red multidireccional en tres sentidos: la territorial, la social y la cultural. 


\section{Contexto y antecedentes del caso}

\subsection{Aproximación al marco territorial}

Para encuadrar los conocimientos físicos y socioeconómicos del territorio que nos ocupa, nos fijamos en su extensión, en su conectividad territorial interna-externa (Tabla 1) y en sus asentamientos históricos, urbanos e industriales (Tabla 1, Figura 1). Ello nos ayuda a entender sus limitadas vinculaciones de movilidad y la dispersión de esos enclaves urbanos. Desde la perspectiva de la disciplina arquitectónica, según expone Muntañola (2011), "El cerebro del arquitecto [...] es la sede capaz de imaginar culturas" (p. 2), luego, por ende, también debe ser capaz de interpretarlas y, en primera instancia, nuestro contacto directo con el lugar debe formar parte de este trabajo. Además, el mismo autor (Muntañola, 2008) se inspira en las teorías de Bajtin (1997) y expone la necesidad de recuperar el diálogo interdisciplinar entre arquitectos y geógrafos para avanzar en el desarrollo creativo y científico de sus obras. Por tanto, para afrontar el reto de nuestras investigaciones y fomentar la interdisciplinariedad, nos alineamos con este ideario.

Tabla 1. Información territorial estadística y de planeamiento

\begin{tabular}{lccccc}
\multicolumn{2}{c}{ Instituto Nacional de Estadística (INE 2011) } & \multicolumn{3}{c}{ Planeamiento (2010) } \\
\hline & término mcpal. & distancia Valencia & núcleo histórico & suelo urbano & suelo industrial \\
& Ha & Km & Ha & Ha & 0,00 \\
\hline Alcublas & 4.351 & 50,00 & 1,97 & 26,76 & 0,00 \\
Alpuente & 13.833 & 87,30 & 2,97 & 20,46 & 0,00 \\
Andilla & 14.278 & 70,50 & 4,06 & 7,68 & 0,00 \\
Aras de los Olmos & 7.604 & 98,00 & 4,24 & 18,92 & 0,00 \\
Benagéber & 6.982 & 109,00 & 0,00 & 7,97 & 4,57 \\
Bugarra & 4.031 & 40,30 & 2,70 & 20,87 & 1,39 \\
Calles & 6.454 & 65,20 & 1,93 & 34,99 & 1,18 \\
Chelva & 19.056 & 69,00 & 13,83 & 34,65 & 1,14 \\
Chulilla & 6.178 & 60,00 & 2,46 & 10,63 & 5,61 \\
Domeño & 6.880 & 34,60 & 0,00 & 28,98 & 6,14 \\
Gestalgar & 6.973 & 46,10 & 5,87 & 17,63 & 0,00 \\
Higueruelas & 1.880 & 59,00 & 6,30 & 28,69 & 16,39 \\
La Yesa & 8.468 & 80,50 & 10,67 & 10,67 & 12,69 \\
Losa del Obispo & 1.217 & 52,00 & 3,34 & 15,34 & 0,00 \\
Pedralba & 5.885 & 35,00 & 2,36 & 30,41 & 3,22 \\
Sot de Chera & 3.875 & 68,00 & 1,80 & 9,53 & 0,00 \\
Titaguas & 6.321 & 88,00 & 9,91 & 22,28 & 55,30 \\
Tuéjar & 12.192 & 73,90 & 12,98 & 39,26 & 125,62 \\
Villar del Arzobispo & 4.070 & 50,00 & 3,48 & 87,66 & 473,38 \\
\hline Total & 140.528 & & 90,87 & &
\end{tabular}


Una plataforma de conocimiento intergeneracional e interdisciplinar en la comarca de La Serranía (Valencia)

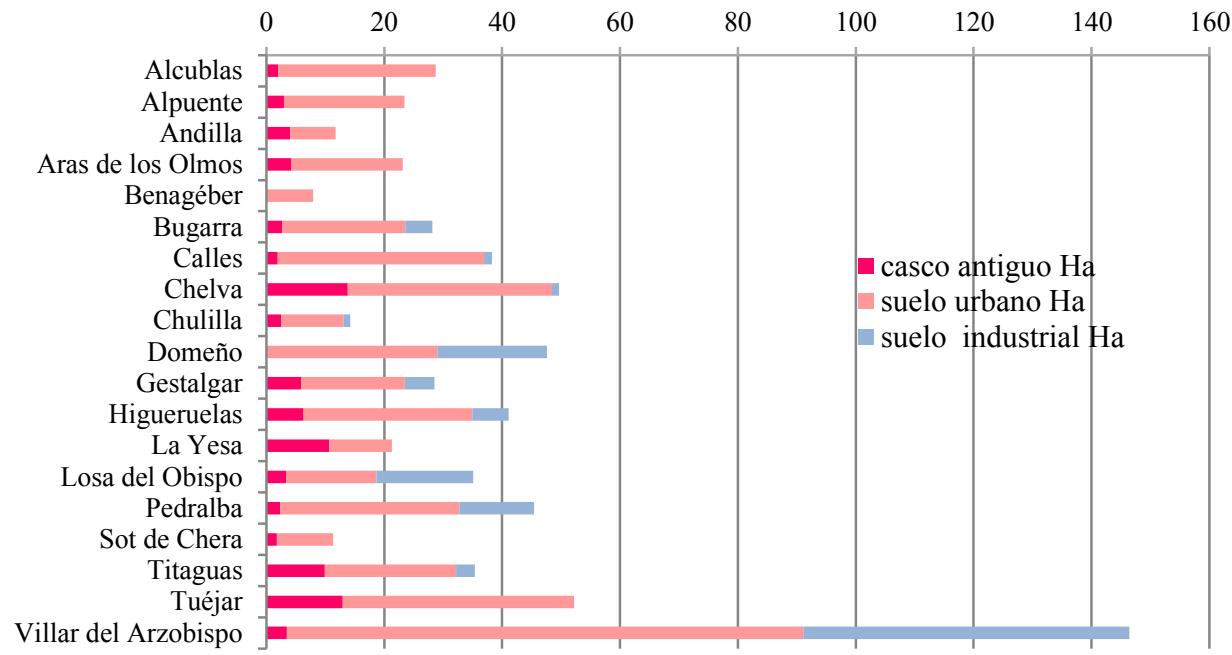

Fig. 1 Gráfico de información territorial de planeamiento (2010)

Fuente: elaboración propia de tabla y gráfico

\subsection{Aproximación al marco social}

Al examinar la situación de su poblamiento, nos detenemos en los resultados demográficos registrados por el INE (1877-2017) y en ellos se detecta la pérdida de su masa humana (Figura 2). De este modo, al combinar estas circunstancias con su situación territorial, nos revela su baja densidad poblacional y su consecuente carencia de servicios como ya nos describía Escalona y Díez (2007) en su estudio de caso sobre los pequeños municipios de áreas rurales. Sin embargo, en La Serranía valenciana, al analizar el registro de sus viviendas de primera y segunda residencia, en base a los datos extraídos del INE (2011) (Figura 3), la población puede multiplicarse si su ocupación es completa y, por tanto, ese déficit de servicios puede agravarse más.

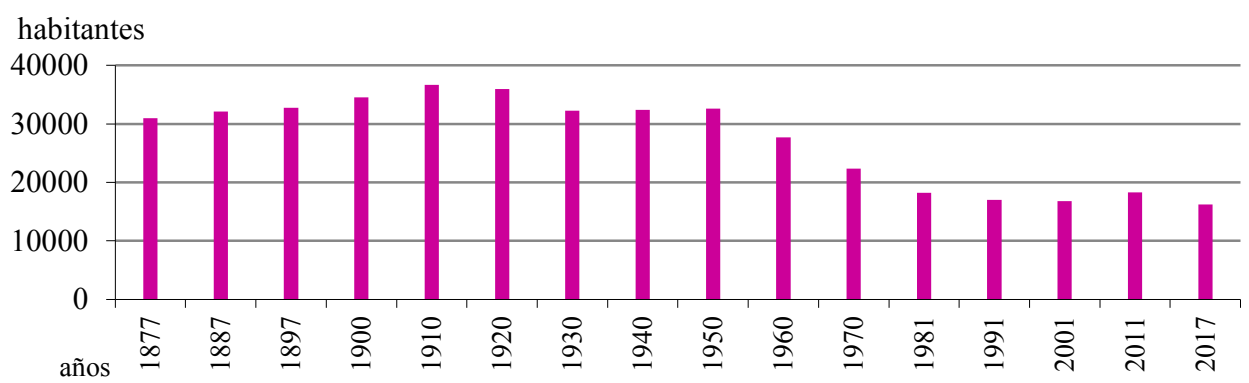

Fig. 2 Población residente en La Serranía entre 1877 y 2017 según INE 


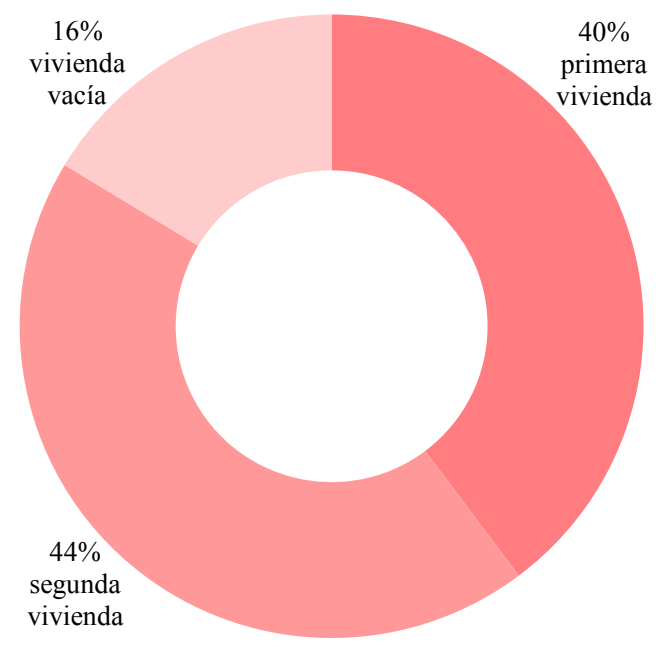

Fig. 3 Gráfico porcentual de primera vivienda, segunda vivienda y vivienda vacía según INE 2011

Fuente: elaboración propia de ambos gráficos

\subsection{Aproximación al marco cultural}

Para analizar el estado de su patrimonio cultural reconocido, estudiamos el Inventario General de Patrimonio (IGPCV) en base a la Ley del Patrimonio Cultural Valenciano (1998) y a nuestros estudios previos desarrollados en esta línea de investigación (Figura 4).

En la zona de estudio, una comarca formada por la agrupación de diecinueve términos con sus correspondientes municipios y aldeas, se descubre la situación contenida de un patrimonio natural y cultural transversal. Es decir, aunque la historia de sus pueblos y civilizaciones se construye a partir de las vivencias de sus moradores, su territorio es único e irremplazable. Luego, en este ámbito rural y disperso, tenemos la oportunidad de establecer una planificación participativa entre sus ciudadanos para cuidar la salud de su patrimonio cultural. 
Una plataforma de conocimiento intergeneracional e interdisciplinar en la comarca de La Serranía (Valencia)

\section{TOTALES}

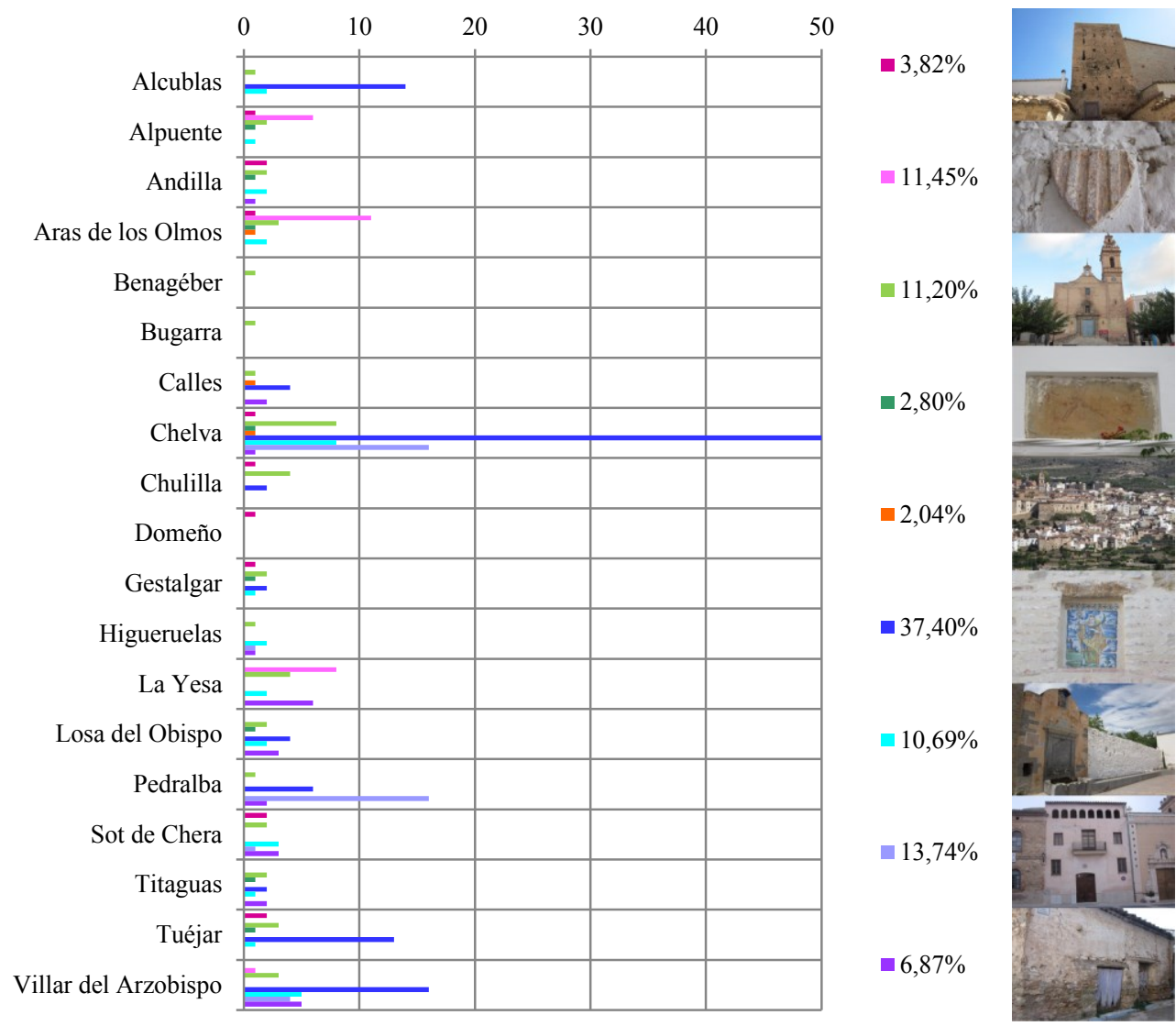

Fig. 4 Gráfico de información patrimonial según IGPCV, planeamiento vigente y estudio propio (Roger, 2015)

Fuente: elaboración propia

\section{Justificación}

El interés profesional por la zona se despierta como una oportunidad al profundizar en la riqueza patrimonial de sus pueblos y aldeas. En ella se descubren vacíos de atención que fijan nuestra investigación, por lo que, a nivel nacional e internacional, el presente planteamiento pretende arrojar luz sobre esa amplia riqueza local de territorios anónimos y de poca repercusión social, dada su escasa presencia de población, con la incorporación de estrategias de participación e interconexión colegio-universidad y territorio-ciudad para 
cultivar la cohesión social de esos pueblos reforzada sobre el aprecio hacia el patrimonio. Es decir, la necesidad de iniciar esta investigación surge porque el estado de conservación y mantenimiento del patrimonio cultural requiere de la participación ciudadana de quienes conviven con ello para garantizar su conocimiento y su reconocimiento pasado, presente y futuro. Se busca la implicación en su entorno cotidiano para contribuir a estimular su sentimiento grupal (Ramón, 2015).

Se detecta que aquello que tenemos más cerca lo conocemos menos, lo estudiamos menos, lo valoramos menos, lo apreciamos menos e incluso lo defendemos menos. Puede parecer una visión pesimista pero para actuar con cautela debemos contemplar ese aspecto negativo porque el ser humano, para ver su valor representado, necesita verse diferenciado respecto a su prójimo e igualmente las sociedades se mueven por esa caracterización distintiva.

Los estudios realizados, tanto dentro de la disciplina artística como de la humanística e incluso de la didáctica, no describen un planteamiento metodológico general de trabajo participativo entre jóvenes y adultos, entre la administración educativa y la gubernamental, entre la sociedad civil y la clase política. Aunque existen trabajos de investigación relacionados con esta temática desde que en 2012 se puso en marcha el Plan Nacional de Educación y Patrimonio (Carrión, 2015), estos no llegan a las zonas más rurales del territorio donde la huella de la intervención humana es precisamente menos relevante. Además, dado el actual estado de globalización, esas zonas están expuestas al riesgo de ser explotadas de manera indiscriminada e incontrolada, por lo que deberemos permanecer atentos y vigilantes de forma permanente. En consecuencia, para garantizar ese control, se alude a los habitantes del territorio por ser sus beneficiarios directos, pero también se incluye a los visitantes, los estudiosos, los investigadores, los profesionales y, cómo no, los políticos.

Para ello, se proponen acciones fundamentadas sobre la interdisciplinariedad, la interculturalidad, la multiterritorialidad y la pluralidad en general, a través de valores de intercambio, colaboración, cooperación, participación y respeto. Para ello se recurre al valor de las redes de comunicación y las nuevas tecnologías sin perder el rumbo de la “formación integral del ciudadano libre del siglo XXI" (Huerta y Domínguez, 2014, p. 22).

\section{Acciones planificadas}

Se valora como prioritaria la revisión y actualización del contenido de los catálogos e inventarios para garantizar la protección del patrimonio cultural en la comarca y se estima fundamental educar a los jóvenes como receptores y trasmisores de la memoria, pero también es necesario refrescar el espíritu de colaboración entre las administraciones y los 
ciudadanos como guardianes de la cultura. Por ello, para aunar ambas intenciones, sobre la ideación de un inventario interactivo (Figura 5), se propone el diseño de una herramienta de documentación, información, gestión y protección a cargo de la administración autonómica y en colaboración continua con las locales; con un programa de revisiones anuales como mecánica de control, comprobación y actualización permanente; y con las medidas de fomento como incentivo a través de ayudas directas, de sistemas de financiación, de beneficios fiscales y de inversiones culturales (Ley 6/1998). Es decir, se propone abrir este proceso a la participación ciudadana desde todos los ámbitos y a través de las siguientes iniciativas educativas, expositivas y constructivas:

$1^{\circ}$. Fase documental: convocatoria de concursos fotográficos, audiovisuales y vídeos, de dibujo y pintura, de maqueta y escultura, de redacción y literatura. Dirigida a niños, adolescentes y mayores en diferentes categorías

$2^{\circ}$. Fase informativa: exposición del material obtenido en los diferentes ámbitos y en convocatoria abierta a la ciudadanía, incluidas visitas de reconocimiento in situ.

$3^{\circ}$. Fase de gestión: tratamiento de los resultados obtenidos en las fases anteriores e incorporación de sus resultados al catálogo interactivo.

$4^{\text {}}$. Fase de protección: realización de talleres de limpieza, mantenimiento y conservación en los lugares y espacios patrimoniales, con grupos de trabajo voluntario dirigidos por especialistas de cada materia.

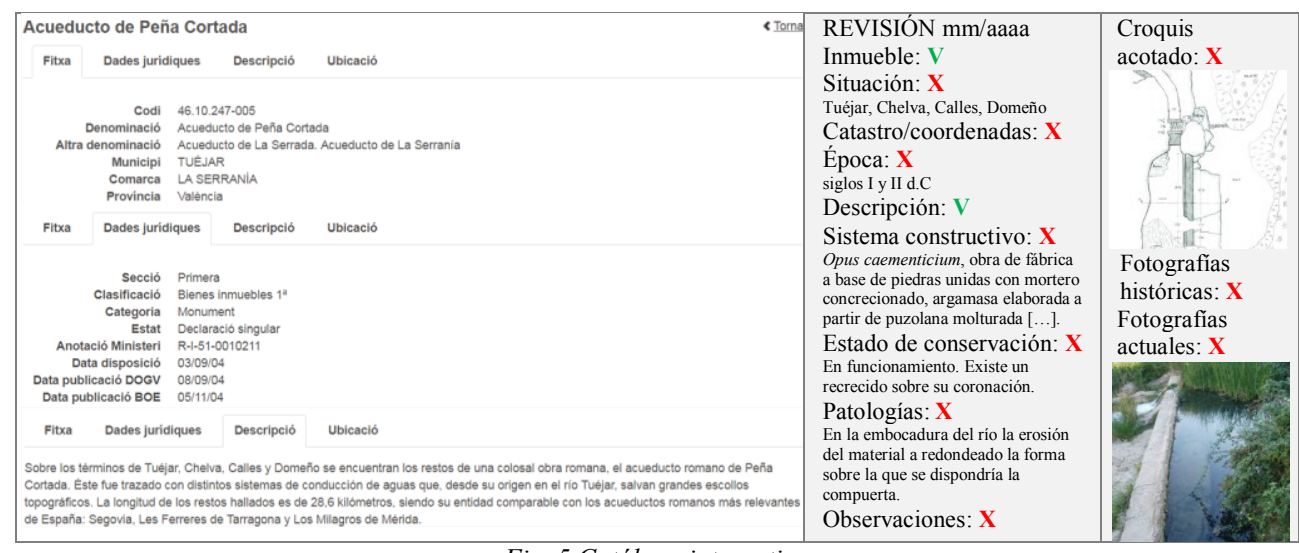

Fig. 5 Catálogo interactivo

Fuente: elaboración propia

Para la justificación de esas revisiones, se definen unos campos de obligada inspección que se cumplimenten con los resultados obtenidos de la selección contrastada y participativa de las anteriores iniciativas educativas, expositivas y constructivas. Se valorarían en positivo (V) los que se mantienen y en negativo (X) los que se modifican o en los que se detectan 
discrepancias a falta de investigar su justificación. Además, se reservaría el espacio de observaciones para proponer acciones, intervenciones y proyectos.

Por otro lado, se plantea la creación de una plataforma cultural participativa en línea desde la que alimentar todos estos campos (Figura 6) para dar continuidad a los trabajos realizados y abrir paso a nuevas líneas de investigación, para documentar los estudios y las actividades desarrolladas, para difundir y dar a conocer las diferentes iniciativas y enfoques y para poner en valor la memoria cultural de esta comarca.

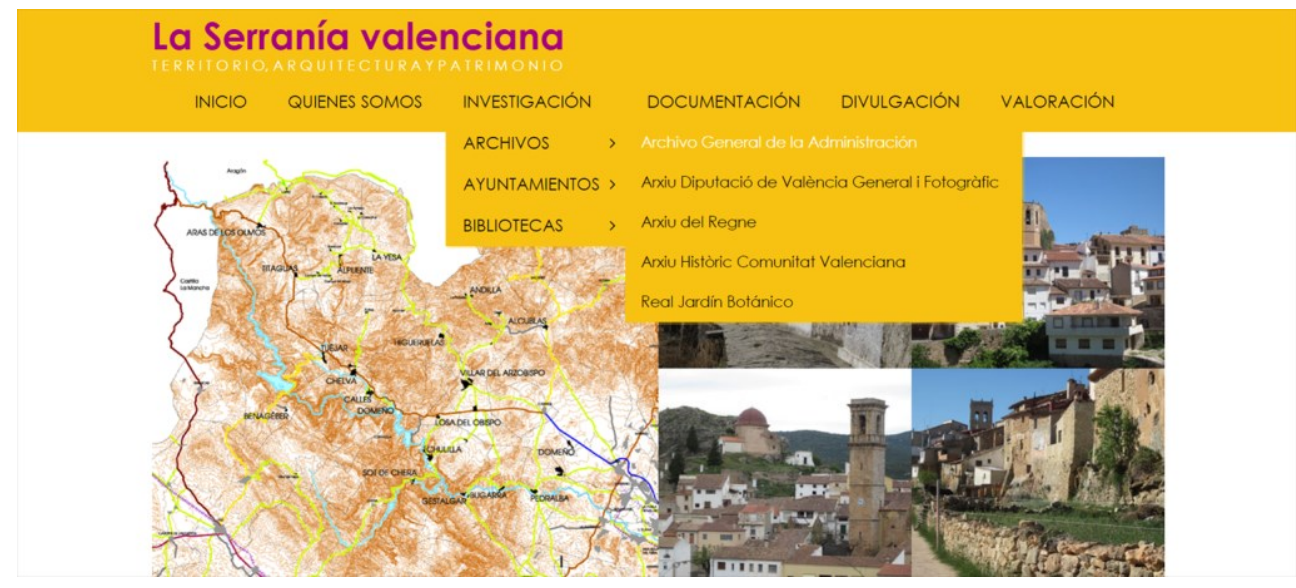

Fig. 6 Plataforma cultural participativa

Fuente: elaboración propia

Para la investigación, se abren itinerarios de búsqueda, a través de los archivos, bibliotecas y fuentes locales, en los que se han localizado referentes y se trata de reconducir esa información dispersa para ponerla al alcance de la sociedad local.

Para la documentación, se prevén enlaces con distintas publicaciones digitales, libros, tesis, capítulos de libro, revistas, artículos, vídeos de entrevistas, reportajes y documentales, cuya calidad y selección se sometería a la revisión por pares ciegos realizada a través de un comité de colaboradores expertos.

Para la divulgación, se ofrece la propia plataforma como espacio expositivo y, a la vez, difusor de los eventos organizados o recomendados y clasificados por materias: pintura, escultura, arquitectura, música, literatura, cine, teatro, previo filtro implementado según los mismos criterios del campo de la documentación. 
Para la valoración, se plantea la organización de debates, mesas redondas, jornadas, seminarios y congresos que posibiliten el diálogo interno y externo entre sus ciudadanos, sus visitantes, sus dirigentes, sus profesionales, sus trabajadores y sus empresarios. Con la inclusión de un apartado destinado a la incorporación de cuestionarios y sondeos que valoren todas las acciones y aporten nuevas perspectivas a la participación colectiva.

\section{Resultados y expectativas}

El presente trabajo aspira a despertar intereses, sinergias, innovaciones y emprendimientos que contribuyan a fortalecer los valores culturales de la comarca de La Serranía y propone ir más allá del espacio virtual para crear un espacio de actividad colaborativa con reaprovechamiento de instalaciones o edificaciones en desuso como modelo ejemplarizante de revalorización patrimonial. Además, dentro de ese ambiente rural anónimo, podría servir para cubrir las deficiencias de equipamientos dotacionales, en cuanto a actividades socioculturales, artesanales o de negocios, al convertirlo en enclave de acogida para asociaciones y entidades sociales, para artistas y artesanos, para innovadores y emprendedores y para técnicos y profesionales.

Estas ideas que se han ido construyendo a lo largo de nuestras investigaciones y que se enmarcan en un entorno territorial, para una población rural y con un bagaje cultural consolidado, anhelan converger en la localización y ser visibles a la globalización. Todas ellas se van engarzando unas con otras y nos unen cada vez más al lugar a la vez que nos proyectan hacia el futuro.

\section{Conclusión}

Como aproximación externa al territorio, me dispongo a situar al alcance de la comunidad comarcal esta plataforma virtual (sitio web) como escenario común de intercambio de conocimientos, de experiencias y de propuestas, a la vez que me propongo iniciar la búsqueda de ese lugar físico de encuentro (sitio coworking) como aproximación interna al territorio, a su sociedad y a sus valores. Pues, aunque estas intenciones se han ido transmitiendo a lo largo de las investigaciones precedentes, desde ahora se pretende darles difusión y comunicación formal. 


\section{Referencias}

Bajtin, M.M. (1997). Hacia una filosofía del acto ético. De los borradores y otros escritos (ca. 1924). [Traducción del ruso de Tatiana Bubnova]. Barcelona: Anthropos.

Carrión, A. (Coord.). (2015). Plan Nacional de Educación y Patrimonio. Secretaría General Técnica. Recuperado de http://ipce.mcu.es/conservacion/planesnacionales/educacion.html

Escalona Orcao, A.I. y Díez Cornago, C. (2007). Despoblación territorial y oferta de servicios: diagnóstico y propuestas. En C. Serrano Lacarra (Coord.), Despoblación y territorio (pp. 61-115). Zaragoza: Centro de Estudios sobre la Despoblación y Desarrollo de Áreas Rurales (CEDDAR).

Generalitat Valenciana. Conselleria de Educación, Investigación, Cultura y Deporte. Inventario General de Patrimonio. Recuperado de http://www.ceice.gva.es/es/web/patrimonio-cultural-ymuseos/inventario-general

Instituto Nacional de Estadística. Censos de Población y Viviendas. Fondo documental del Instituto nacional de Estadística (1877-2017). Población, superficie y densidad por municipios (2011). Población vinculada y sus componentes (2011). Recuperado de http://www.ine.es/

Huerta, R. y Domínguez, R. (2014). Investigar en educación artística: nuevos entornos y retos pendientes. Educación Artística Revista de Investigación, 5, 11-22. http://dx.doi.org/10.7203/eari.5.4139

Ley 4/1998, de 11 de junio, del Patrimonio Cultural Valenciano. Diari Oficial de la Generalitat Valenciana (18 de junio de 1998), núm. 3267. Boletín Oficial del Estado. Valencia, 22 de julio de 1998, núm. 174.

Muntañola Thornberg, J. (2011). El diálogo entre proyecto y lugar. Un reto para la arquitectura del siglo XXI. Cuadernos de Proyectos Arquitectónicos. "El lugar”, 2, 33-38.

Muntañola Thornberg, J. (2008). Geógrafos y arquitectos; nuevos retos y viejos problemas. En Scripta Nova, Revista electrónica de Geografía y Ciencias Sociales, XII, 270, 147.

Oficina de información urbanística del Colegio Territorial de Arquitectos de Valencia. Planeamiento vigente y en tramitación. Recuperado de http://oiu.ctav.es/Planeamiento/Buscar

Ramón, R. (2015). Reinventar la ciudad, desarrollo de las competencias básicas a través de un proyecto de creación urbana. Revista Matèria-Prima, 3 (2), 79-88.

Roger Espinosa, F. (2015). Núcleos históricos tradicionales en la comarca de La Serranía. (Tesis doctoral). Universitat Politècnica de València, Valencia. 